PROCEEDINGS OF THE

AMERICAN MATHEMATICAL SOCIETY

Volume 30, No. 1, September 1971

\title{
EMBEDDING THE DUAL OF $\Pi_{m}$ IN THE LATTICE OF EQUATIONAL CLASSES OF COMMUTATIVE SEMIGROUPS
}

\author{
STANLEY BURRIS ${ }^{1}$ AND EVELYN NELSON ${ }^{2}$
}

Abstract. The lattice of equational classes of commutative semigroups does not satisfy any special lattice laws.

In [4] R. Schwabauer proved that the lattice $\mathscr{L}$ of equational classes of commutative semigroups is nonmodular. In this paper we will prove a maximal extension of this result, namely, $\&$ does not satisfy any special lattice laws.

The free commutative semigroup on countably many generators, $\mathfrak{F}(\omega)$, is the set of sequences $\left(u_{n}\right)_{n \in N}$ of nonnegative integers, such that $u_{n}=0$ for all but finitely many $n \in N$ and $\sum u_{n} \geqq 1$, with component-wise addition. [For convenience, we write $\left(u_{n}\right)$ for $\left(u_{n}\right)_{n \in N}$.]

A commutative semigroup equation is a pair $\left(\left(u_{n}\right),\left(v_{n}\right)\right)$ of elements of $\mathfrak{F}(\omega)$ (see [1]). A commutative semigroup $(\mathcal{S}, \cdot)$ satisfies the equation $\left(\left(u_{n}\right),\left(v_{n}\right)\right)$ iff, for every family $\left(a_{n}\right)$ of elements of $\delta$, $\Pi\left\{a_{n}^{u_{n}} \mid u_{n} \neq 0\right\}=\Pi\left\{a_{n}^{v_{n}} \mid v_{n} \neq 0\right\}$.

A set $\Sigma$ of equations is closed [1, p. 170, Definition 2] iff it contains all trivial equations, is symmetric and transitive, and is closed under multiplication and substitution of terms for variables. Thus $\Sigma$ is closed iff it satisfies the following conditions:

(P1): $\left(\left(u_{n}\right),\left(u_{n}\right)\right) \in \Sigma$ for all $\left(u_{n}\right) \in \mathfrak{F}(\omega)$.

(P2): If $\left(\left(u_{n}\right),\left(v_{n}\right)\right) \in \Sigma$ then $\left(\left(v_{n}\right),\left(u_{n}\right)\right) \in \Sigma$.

(P3): If $\left(\left(u_{n}\right),\left(v_{n}\right)\right) \in \Sigma$ and $\left(\left(v_{n}\right),\left(w_{n}\right)\right) \in \Sigma$ then $\left(\left(u_{n}\right),\left(w_{n}\right)\right) \in \Sigma$.

(P4): If $\left(\left(u_{n}\right),\left(v_{n}\right)\right) \in \Sigma$ and $\left(\left(u_{n}^{\prime}\right),\left(v_{n}^{\prime}\right)\right) \in \Sigma$ then $\left(\left(u_{n}+u_{n}^{\prime}\right)\right.$, $\left.\left(v_{n}+v_{n}^{\prime}\right)\right) \in \Sigma$.

(P5): If $\left(\left(u_{n}\right),\left(v_{n}\right)\right) \in \Sigma,\left(k_{n}\right) \in \mathfrak{F}(\omega)$ and $p \in N$, then the result of "substituting $\left(k_{n}\right)$ for the $p$ th variable" in $\left(\left(u_{n}\right),\left(v_{n}\right)\right)$ is in $\Sigma$, i.e., $\left(\left(w_{n}\right),\left(x_{n}\right)\right) \in \Sigma$ where $w_{n}=u_{n}+k_{n} u_{p}$ for $n \neq p, w_{p}=k_{p} u_{p}$, and $x_{n}=v_{n}$ $+k_{n} v_{p}$ for $n \neq p$ and $x_{p}=k_{p} v_{p}$.

(Note that these conditions (P1) to (P5) are, essentially, a restatement of conditions (i) to (v) in Grätzer [1, p. 170, Definition 2]; in condition (iv) we need only consider the one binary operation, hence

Received by the editors May 14, 1970.

AMS 1969 subject classifications. Primary 2090, 0630; Secondary 0830.

Key words and phrases. Equation, equational class, commutative semigroup, lattice, partition lattice, lattice law.

${ }^{1}$ Research supported by NRC Grant A7256.

${ }^{2}$ Research supported by NRC Grant A2985.

Copyright (C) 1971, American Mathematical Society 
the different form of $(\mathrm{P} 4).) \Gamma(\Sigma)$ will denote the deductive closure of $\Sigma$.

Let $\mathscr{L}^{\prime}$ be the lattice of closed sets of commutative semigroup equations; then $\mathscr{L}^{\prime}$ is dually isomorphic to the lattice $\mathscr{L}$ of equational classes of commutative semigroups.

For each $m \in N$, let $\Pi_{m}$ be the partition lattice on $\{1,2, \cdots, m\}$.

THEOREM 1. For each $m \in N, \Pi_{m}$ is (isomorphic to) a sublattice of $\mathfrak{L}^{\prime}$.

Proof. Let $m$ be a fixed natural number. If $\pi \in \Pi_{m}$, we write $\equiv_{\pi}$ for the equivalence relation on $\{1,2, \cdots, m\}$ induced by $\pi$. Let $\Sigma=\left\{\left(\left(u_{n}\right),\left(v_{n}\right)\right) \mid \sum u_{n}, \sum v_{n} \geqq 2 m+2\right\} \cup\left\{\left(\left(u_{n}\right),\left(u_{n}\right)\right) \mid\left(u_{n}\right) \in \mathfrak{F}(\omega)\right\}$. It is clear from (P1) to (P5) that $\Sigma$ is a closed set of equations. For each $\pi \in \Pi_{m}$, define a set $\Sigma(\pi)$ of equations as follows: $\left(\left(u_{n}\right),\left(v_{n}\right)\right)$ $\in \Sigma(\pi)$ iff there exist $j, k$ with $u_{n}=v_{n}=0$ for all $n \neq j, k, u_{j}+u_{k}=2 m$ $+1=v_{j}+v_{k}$, and either $u_{j} \equiv_{\pi} v_{j}$ or $u_{k} \equiv_{\pi} v_{k}$. Note that if $u_{j}+u_{k}=2 m+1$ and $u_{j} \equiv_{\pi} v_{j}$ then $u_{k}>m$, thus $u_{k}$ is not equivalent to anything modulo $\pi$. Then, since $\pi$ is a partition, it follows that if $\left(\left(u_{n}\right),\left(v_{n}\right)\right) \in \Sigma(\pi)$ and if $\left(\left(v_{n}\right),\left(w_{n}\right)\right) \in \Sigma(\pi)$, then $\left(\left(u_{n}\right),\left(w_{n}\right)\right) \in \Sigma(\pi) . \Sigma(\pi)$ is obviously symmetric. Applying (P4) to two equations in $\Sigma(\pi)$ yields an equation in $\Sigma$. Applying (P5) with $\sum k_{n}=1$ to an equation in $\Sigma(\pi)$ yields either a trivial equation or an equation in $\Sigma(\pi)$; applying (P5) with $\sum k_{n}$ $\geqq 2$ and with $u_{p} \geqq 1$ yields an equation in $\Sigma$; and applying (P5) with $u_{p}=0$ does not change the equation. Thus $\Sigma \cup \Sigma(\pi)$ is a closed set of equations.

For two partitions $\pi_{1}, \pi_{2}$, if $\pi_{1} \wedge \pi_{2}$ and $\pi_{1} \bigvee \pi_{2}$ are the meet and join of $\pi_{1}$ and $\pi_{2}$ in $\Pi_{m}$ then

$\left(\Sigma \cup \Sigma\left(\pi_{1}\right)\right) \cap\left(\Sigma \cup \Sigma\left(\pi_{2}\right)\right)=\Sigma \cup\left(\Sigma\left(\pi_{1}\right) \cap \Sigma\left(\pi_{2}\right)\right)=\Sigma \cup\left(\Sigma\left(\pi_{1} \wedge \pi_{2}\right)\right)$.

Also it is clear that

$$
\begin{aligned}
\left(\Sigma \cup \Sigma\left(\pi_{1}\right)\right) \bigvee \mathfrak{L}^{\prime}\left(\Sigma \cup \Sigma\left(\pi_{2}\right)\right) & =\Gamma\left(\Sigma \cup \Sigma\left(\pi_{1}\right) \cup \Sigma\left(\pi_{2}\right)\right) \\
& \subseteq \Sigma \cup \Sigma\left(\pi_{1} \vee \pi_{2}\right) .
\end{aligned}
$$

Conversely, if $\left(\left(u_{n}\right),\left(v_{n}\right)\right) \in \Sigma\left(\pi_{1} \bigvee \pi_{2}\right)$, then there exist $j, k$ with $u_{n}=v_{n}=0$ for all $n \neq j, k, u_{k}+u_{j}=v_{k}+v_{j}=2 m+1$ and, w.l.o.g. $u_{j}$ $\equiv_{\pi_{1} \vee \pi_{2}} v_{j}$. But then there exist $w_{1}, \cdots, w_{p}$ in $\{1,2, \cdots, m\}$ such that $w_{1}=u_{j}, w_{p}=v_{j}$, and $w_{i} \equiv_{\pi_{1}} w_{i+1}$ for $i$ odd, $w_{i} \equiv_{\pi_{2}} w_{i+1}$ for $i$ even. Let $\alpha_{i} \in \mathfrak{F}(\omega)$ have $j$ th entry $w_{i}$, $k$ th entry $2 m+1-w_{i}$, and all other entries zero. Then $\alpha_{1}=\left(u_{n}\right), \alpha_{p}=\left(v_{n}\right)$ and $\left(\alpha_{i}, \alpha_{i+1}\right) \in \Sigma\left(\pi_{1}\right)$ for $i$ odd and $\left(\alpha_{i}, \alpha_{i+1}\right) \in \Sigma\left(\pi_{2}\right)$ for $i$ even. It follows that

$$
\left(\left(u_{n}\right),\left(v_{n}\right)\right)=\left(\alpha_{1}, \alpha_{p}\right) \in \Gamma\left(\Sigma\left(\pi_{1}\right) \cup \Sigma\left(\pi_{2}\right)\right) .
$$

Hence 
1971]

$$
\Sigma \cup \Sigma\left(\pi_{1} \bigvee \pi_{2}\right) \subseteq \Gamma\left(\Sigma\left(\pi_{1}\right) \cup \Sigma\left(\pi_{2}\right) \cup \Sigma\right)
$$

Thus we have

$$
\left(\Sigma \cup \Sigma\left(\pi_{1}\right)\right) \wedge \mathcal{L}^{\prime}\left(\Sigma \cup \Sigma\left(\pi_{2}\right)\right)=\Sigma \cup \Sigma\left(\pi_{1} \wedge \pi_{2}\right)
$$

and

$$
\left(\Sigma \cup \Sigma\left(\pi_{1}\right)\right) \vee \mathfrak{L}^{\prime}\left(\Sigma \cup \Sigma\left(\pi_{2}\right)\right)=\Sigma \cup \Sigma\left(\pi_{1} \vee \pi_{2}\right)
$$

If follows that the mapping $\pi \rightarrow \Sigma \cup \Sigma(\pi)$ is a homomorphism of $\Pi_{m}$ into $\mathfrak{L}^{\prime}$.

It is clear that if $\pi_{1} \neq \pi_{2}$ then $\Sigma \cup \Sigma\left(\pi_{1}\right) \neq \Sigma \cup \Sigma\left(\pi_{2}\right)$; thus this homomorphism is one-to-one, and this yields the desired result.

THEOREM 2. $\&$ does not satisfy any special lattice laws.

Proof. From D. Sachs [3] it is known that the family of partition lattices $\Pi_{m}, m=1,2, \cdots$, does not satisfy any special lattice laws.

CONCLUDING REMARK. One might consider the possibility of embedding the dual of $\Pi_{\infty}$ into $\mathscr{L}$, but a recent paper of P. Perkins [2] shows that this is impossible because $\mathscr{L}$ is countable, whereas $\Pi_{\infty}$ is uncountable.

\section{REFERENCES}

1. G. Grätzer, Universal algebra, Van Nostrand, Princeton, N. J., 1968. MR 40 \#1320.

2. P. Perkins, Bases for equational theories of semigroups, J. Algebra 11 (1969), 298-314. MR 38 \#2232.

3. D. Sachs, Identities in finite partition lattices, Proc. Amer. Math. Soc. 12 (1961), 944-945. MR 24 \#A3101.

4. R. Schwabauer, A note on commutative semigroups, Proc. Amer. Math. Soc. 20 (1969), 503-504. MR 38 \#2233.

University of Waterloo, Waterloo, Ontario, Canada

McMaster University, Hamilton, Ontario, Canada 